\title{
Isolation of novel bovine parainfluenza virus type 5 (bPIV5) and its incidence in Korean cattle
}

\author{
Dong-Kun Yang*, Jin-Ju Nah, Ha-Hyun Kim, Sung-Suk Choi, You-Chan Bae, Jung-Won Park, Jae-Young Song \\ Animal and Plant Quarantine Agency, Ministry of Agriculture, Food and Rural Affairs, Anyang 430-757, Korea
}

(Received: February 7, 2014; Revised: March 28, 2014; Accepted: May 23, 2014)

\begin{abstract}
Four viruses showing cytopathic effects in MDBK cells were isolated from brains of cattle showing downer cattle syndrome in 2012. The isolates were confirmed to belong to the genus Rubulavirus of the subfamily Paramyxovirinae. Isolate QIA-B1201 had the ability to hemagglutinate red blood cells from several species of animals and was capable of adsorbing guinea pig erythrocytes on the surface of infected Vero cells. Nucleotide sequence analysis showed that two isolates (QIA-B1201 and QIA-B1204) had high similarity with other human and animal PIV5 isolates ranging from 98.1 to $99.8 \%$. The highest sequence similarity of the two isolates corresponded to strain KNU-11 (99.8\% at the nucleotide and amino acid level) isolated from suckling piglets in Korea in 2012. To evaluate the virulence of strain QIA-B1201, we inoculated bPIV5 into 5 week-old mice via both the intraperitoneal and intracranial route. Body weight was not significantly altered in mice inoculated with QIA-B1201. In this study, we isolated and characterized novel bPIV5s from brain samples showing downer cattle syndrome, but were not able to elucidate the pathogenicity of the bPIV $5 \mathrm{~s}$ in mice.
\end{abstract}

Keywords : bovine, neurologic disease, parainfluenza virus 5, virus isolation

\section{Introduction}

It is well known that Paramyxoviruses involve in both respiratory and neurotropic pathogen in a variety of animals and human. The Paramyxoviridae family including important animal pathogens such as bovine respiratory syncytial virus (BRSV) and Newcastle disease (NDV) is classified into two subfamilies; Paramyxovirinae, and Pneumovirinae. The Paramyxovirinae currently consists of five genera, respirovirus, rubulavirus, avularvirus, morbillivirus and henipavirus. Parainfluenza virus 5 (PIV5) previously named as simian virus 5 (SV5) belongs to the genus rubulavirus of the subfamily Paramyxovirinae [11, 16]. The PIV5 contains nonsegmented negative strand RNA genome of approximately 15,000 nucleotides and composes of seven genes, but encodes eight proteins such as nucleocapsid (NC), phospho $(\mathrm{P})$, matrix $(\mathrm{M})$, fusion $(\mathrm{F})$, small hydrophobic $(\mathrm{SH})$, hemagglutinin-neuraminidase $(\mathrm{HN})$ and large protein $(\mathrm{L})$. The $\mathrm{NC}$, $\mathrm{P}$ and $\mathrm{L}$ proteins have a relation to transcription and replication of the viral RNA genome [17]. The V protein involves in viral pathogenesis and $\mathrm{F}$ protein mediates cell fusion that plays a key role entering into cells. HN protein, glycoprotein, is associated with viral entry and release from the host cells. The $\mathrm{M}$ protein plays a pivotal role in virus assembly and budding [15]. The SH protein, a membrane protein, is responsible for blocking TNF- $\alpha$ signaling [19].

The PIV5 previously known as SV5 was first isolated in 1956 from non-inoculated rhesus and cynomolgus monkey kidney cell cultures [9]. Very little attention was paid to this virus until the discovery of the hemadsorption (HAD) method in 1957. Human PIV5 has been isolated from various clinical cases and the neutralizing antibody against PIV5 in non-symptomatic human was reported [5, 10]. Canine parainfluenza virus 5 (cPIV5) renamed by International Committee on Taxonomy of Viruses in 2009 has caused pathological changes of bronchitis and bronchiolitis in combination with bacteria and has been considered as one of the important viral agents causing canine respiratory disease $[1,2]$. It is also noted that the cPIV5 caused acute encephalitis in experimentally infected gnotobiotic dogs [8]. Two porcine PIV5 (pPIV5) have been isolated from the lung of fetus of sow infected with porcine reproductive and respiratory syndrome virus (PRRSV) and piglet in porcine macrophage cells $[7,13]$. Lee et al. [13] reported that the pPIV5 may be one of very mild pathogens to pigs. Several species of animals such as cats, hamster and guinea pigs may be infected with PIV5 [8]. In our laboratory, bovine PIV5 (bPIV5) was recently isolated from the brain of cattle showing neurologic symptoms

*Corresponding author

Tel: +82-31-467-1783, Fax: +82-31-467-1797

E-mail: yangdk@korea.kr 
using the Madin-Darby bovine kidney (MDBK) cells. In this study, to understand the significance of bPIV5, we carried out biologic/molecular characterization of bPIV5 isolates (QIAB1201, QIA-B1204) and surveyed its incidence in Korean cattle.

\section{Materials and Methods}

\section{Sample preparation}

Bovine brain samples were collected from 70 cattle of 17 farms associated with neurologic symptoms including hind limb paralysis in Gyeonggi Province of Korea in 2012. In total, 532 neuro-associated samples including cerebrum, cerebellum, brainstem, sciatic nerve, thoracic nerve and lumbar nerve were grounded with sea sand (Sigma, USA) and then diluted with culture medium into 10 -fold ( $\mathrm{vol} / \mathrm{vol})$. After then, the samples were centrifuged at $3,000 \times \mathrm{g}$ for $10 \mathrm{~min}$. Supernatants were used for the virus isolation.

\section{Virus isolation, hemagglutination assay (HA) and HAD}

Brain samples were inoculated into MDBK and Vero cells, respectively and the cells undergone to blind passages twice. For the identification of unknown virus, the isolated viruses were inoculated into the two kinds of cells grown in alpha minimum essential medium ( $\alpha$-MEM; Gibco-BRL, USA) with $10 \%$ heat-inactivated fetal bovine serum (Gibco-BRL). The cells were incubated in $\mathrm{CO}_{2}$ incubator and observed for 7 days. Murine neuroblastoma NG108-15 cells (ATCC HB12317) grown in DMEM were used to check the biological characterization of the isolates. HA test was performed by preparing serial two fold dilutions of bPIV5, QIA-B1201 into $50 \mathrm{~mL}$ of phosphate buffer saline (PBS, pH 7.2) and with 50 $\mathrm{mL}$ of $0.6 \%$ several kinds of animal erythrocytes. The HA titer was expressed as the reciprocal of the highest dilution of bPIV5 showing HA. For the HAD test, Vero cells inoculated with the isolates were grown for 5 days. After throwing supernatant solution away, the cells were washed with PBS $(\mathrm{pH}$ 7.2) and incubated with red blood cells of guinea pig at $37^{\circ} \mathrm{C}$ for $1 \mathrm{~h}$. After washing in PBS, the cells were examined by microscope to find out the ability of HAD.

\section{Electron microscope}

The MDBK cells inoculated with the isolate were harvested using a rubber policeman at $96 \mathrm{~h}$ post inoculation. After centrifugation of the cells, cell pellet was fixed with
$2.5 \%$ glutaraldehyde in PBS at $4^{\circ} \mathrm{C}$ for $2.5 \mathrm{~h}$ and then fixed with $1 \%$ osmium tetroxide in PBS for $2 \mathrm{hr}$ again. After dehydration, the fixed cells were embedded in resin and ultrathin sections of the cells were made. The sections were stained with uranyl acetate and lead citrate and observed under electronic microscope (H-7100FA; Hitachi, Japan).

\section{RNA extraction and RT-PCR}

Viral RNA was extracted from the four isolates (QIAB1201 QIA-B1204) using an RNA extraction kit (Qiagen, Germany) according to the manufacturer's instructions. The extracted RNA was eluted in $50 \mu \mathrm{L}$ of RNase- and DNasefree water. The first RT-PCR was carried out to screen bPIV5 in brain samples using parainfluenza virus detection kit (iNtRON Biotechnology, Korea). The other RT-PCR was performed with specific primers (PIV5F1, PIV5R1, PIV5F2, PIV5R2, PIV5F3 and PIV5R3) that amplify the F gene of bPIV5 (Table 1). The first RT-PCR was carried out according to manufacturer's indication. The other RT-PCR was performed in a reaction mixture containing $10 \mu \mathrm{L}$ of denatured RNA, $1 \mu \mathrm{L}$ of each primer ( $50 \mathrm{pmol}), 10 \mu \mathrm{L}$ of $5 \times$ buffer (12.5 mM MgCl $), 2 \mu \mathrm{L}$ of dNTP mix, $2 \mu \mathrm{L}$ of enzyme mix (reverse transcriptase and Taq polymerase), and $24 \mu \mathrm{L}$ of distilled water (Qiagen). The cycling profile consisted of cDNA synthesis at $42^{\circ} \mathrm{C}$ for $30 \mathrm{~min}$, followed by 35 cycles of $95^{\circ} \mathrm{C}$ for $30 \mathrm{sec}, 52^{\circ} \mathrm{C}$ for $30 \mathrm{sec}$, and $72^{\circ} \mathrm{C}$ for $45 \mathrm{sec}$, with a final extension at $72^{\circ} \mathrm{C}$ for $5 \mathrm{~min}$. The PCR products were visualized using electrophoresis on $1.8 \%$ agarose gels containing ethidium bromide.

\section{Cloning and sequencing}

For the cloning of F gene, all of the PCR products that were purified using the gel extraction kit were ligated with pGEM-T easy vector (Promega, USA) according to the manufacturer's protocol. Plasmid DNA was isolated from ampli-

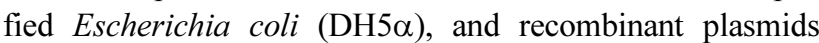
were identified using EcoRI enzyme digestion (Bioneer, Korea). The sequences of the purified plasmids were analyzed using an MJ Research PTC-225 Peltier Thermal Cycler and ABI PRISM BigDye Terminator Cycle Sequencing kits with AmpliTaq DNA polymerase (FS enzyme; Applied Biosystems, USA) according to the manufacturers' protocols. Single-pass sequencing was performed for each template using universal primers (e.g., SP6 and T7). The fluorescent-

Table 1. Oligonucleotide primers used for real-time polymerase chain reaction (RT-PCR) against $\mathrm{F}$ gene of parainfluenza virus 5

\begin{tabular}{ccc}
\hline \hline Primer name & \multicolumn{1}{c}{ Oligonucleotides sequence (5’-3') } & Size of amplicon \\
\hline PIV5F1 & ACC TGG AAT CAC CAG CTT GAT TTA T & \multirow{2}{*}{$810(\mathrm{bp})$} \\
PIV5R1 & TTT CGA CCA CAG TCG GCA AGG TAC T \\
PIV5F2 & CAG CAA TTA CAG CAG CCA ATT GTA A & \\
PIV5R2 & TGC ACT TGC ATA ACA TCG ACC TGC A & $630(\mathrm{bp})$ \\
PIV5F3 & AAA TTG CAG GTC GAT GT ATG CAA G & \\
PIV5R3 & CGA GAC GGT TCT TTC AAT ACT AGT T & $594(\mathrm{bp})$ \\
\hline
\end{tabular}


labeled fragments were purified from the unincorporated terminators using an ethanol precipitation protocol. The samples were resuspended in distilled water and subjected to electrophoresis in an ABI 3730xl sequencer (Applied Biosystems). Both DNA strands were sequenced to verify the sequences.

\section{Phylogenetic analysis}

The nucleotide sequences, accession numbers, and names of the strains used for the phylogenetic analysis in this study were obtained from the GenBank database. The full nucleotide sequence of $\mathrm{F}$ gene of the isolate was compared with that of the other known PIV5 strains using ClustalW2 [12]. Genetic distances were calculated using the Kimura-2 correction parameter and phylogenetic tree was constructed using the neighbor-joining method with 1,000 bootstrap replicates in MEGA4 [18].

\section{Mouse inoculation test}

To investigate the pathogenicity of the bPIV5 isolate, 5 week-old ICR mice were divided into three groups of six female animals. Group 1 was inoculated by intracranial (IC) route with $0.03 \mathrm{~mL}$ of QIA-B1201 $\left(10^{7.0} \mathrm{TCID}_{50} / \mathrm{mL}\right)$ and group 2 by intraperitoneal (IP) route with $0.5 \mathrm{~mL}$ of QIA$\mathrm{B} 1201\left(10^{7.0} \mathrm{TCID}_{50} / \mathrm{mL}\right)$ respectively and group 3 remained as control. Body weight of the mice was measured and clinical signs of the mice were observed for 15 days.

\section{Results}

\section{Identification of bovine PIV5 by biological character- ization}

Each MDBK and Vero cell inoculated with bovine brain samples was examined under microscope for 7 days and blind passage of the samples was conducted. After second passage, mild CPE in MDBK cell was observed, but any great change could not be identified in Vero cells. Finally, four viruses were isolated from 532 bovine brain samples of 70 cattle raised in Gyeonggi-Province of Korea. The three viruses designated as QIA-B1201, QIA-B1202 and QIAB1203 were isolated from the identical farm located in Pocheon district, and the other virus designated as QIAB1204 was isolated other farm located in the same region. To check the biological characterization the four isolates were inoculated into MDBK, Vero and NG108-15 cells. The three kinds of cells inoculated with the four isolates showed similar CPEs in each cell. MDBK and Vero cells inoculated with the QIA-B1201 strain showed weak CPE (Figs. 1A and B), which was characterized by cell rounding, the aggregation and a little detachment of cells, but NG108-15 cells inoculated with the isolate revealed much more distinctive CPE within 3 days post inoculation (DPI) (Fig. 1C). To determine whether the isolates have the ability to HAD, HAD assay was performed on infected Vero cells at 3 DPI. As shown in Fig. 1D, many red blood cells of guinea pig were attached to
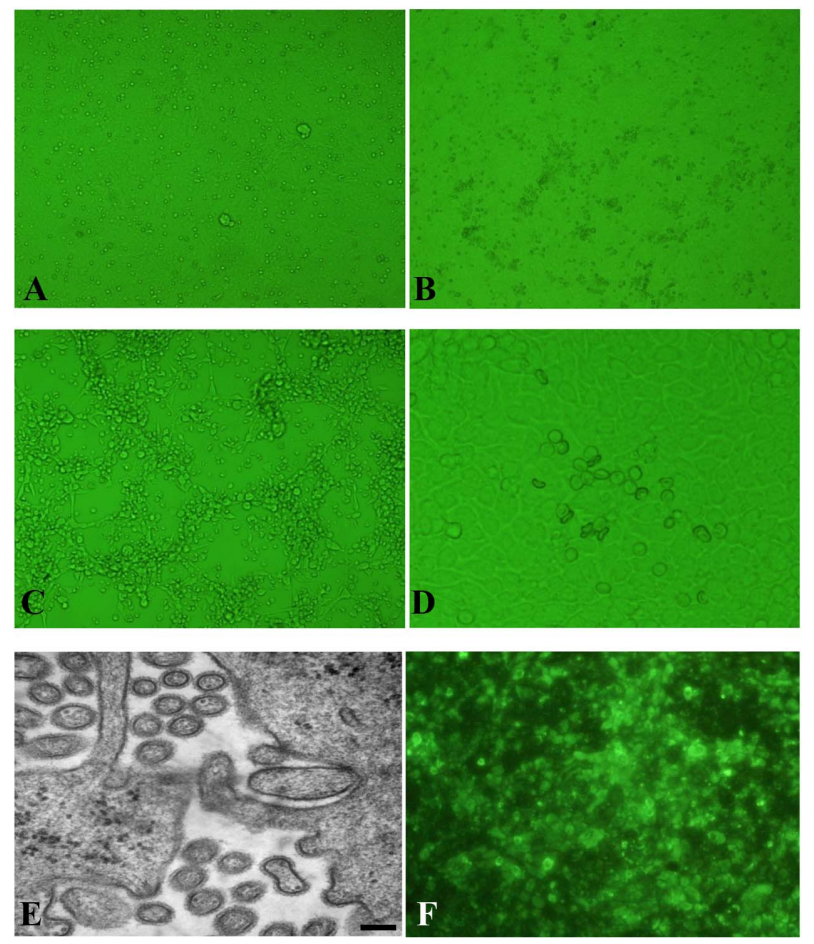

Fig. 1. Cytopathic effects of the Madin-Darby bovine kidney (MDBK) (A), vero (B) and NG108-15 (C) cells infected with the QIA-B1201 strain at 5 days post inoculation. (D) Guinea pig erythrocytes were attached on the infected vero cells. (E) Observation of virus particles ranging from 80 to $150 \mathrm{~nm}$ in size in MDBK cells infected with theisolate. Scale bar $=100 \mathrm{~nm}$. (F) Immunofluorescence in MDBK cells infected with the isolates using the parainfluenza virus type 5 (PIV5) specific antibodies.

the cell surface. In order to check the ability to HA, HA assay was also carried out using supernatant obtained from the infected MDBK cells. All isolates agglutinated erythrocytes of guinea pig, chicken, mouse and goose at $4^{\circ} \mathrm{C}$ or room temperature (Table 2). Virus particles were pleomorphic and varied from $80 \mathrm{~nm}$ to $150 \mathrm{~nm}$ in size. Some viral particles budding on the cell surface were observed and virions cut in longitudinal section were also shown in Fig. 1E. The MDBK cells infected with the isolate were stained with PIV5 specific antibody. As shown in Fig. 1F, fluorescence was noticed in the cytoplasm.

\section{Identification of bovine PIV5 by molecular character- ization}

Even though biologic characterization could provide possible clue, molecular method was applied to get definitive answer of bPIV5. The first RT-PCR was carried out to screen parainfluenza viral RNA in 532 bovine brain samples of 70 cattle in 14 farms. Twelve $(2.25 \%)$ of 532 samples were found to be positive against PIV5. The other RT-PCR using specific primers for PIV5 could amplify three partial F genes from the isolates (Table 1). As shown in Fig. 2, three PCR products of the F gene of isolate (QIA-B1201) were detected 
Table 2. Hemagglutination assay (HA) titer of the isolate according to erythrocytes of animal species

\begin{tabular}{lcc}
\hline \hline Species of animals & HA titer & Condition \\
\hline mouse & 64 & At $4^{\circ} \mathrm{C}$ or $22^{\circ} \mathrm{C}$ \\
Guinea pig & 128 & $0.6 \%$ of red blood cells \\
Chicken & 512 & in PBS (pH 7.0) \\
Goose & 128 & \\
Pig & 64 & \\
\hline
\end{tabular}

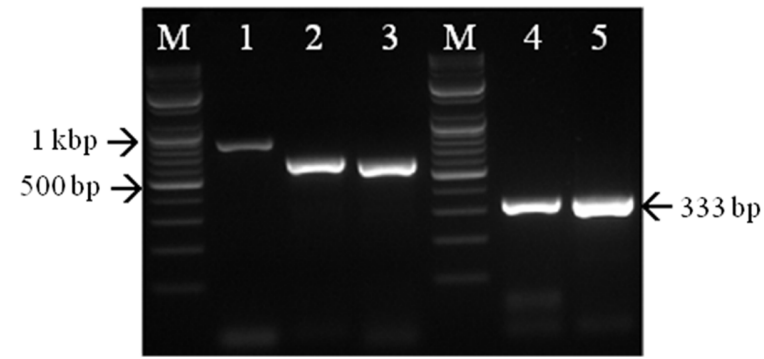

Fig. 2. Three specific primer sets targeted $\mathrm{F}$ gene of the QIAB1201 strain were used for RT-PCR and RT-PCR kit was applied to detect parainfluenza viral RNA in the same strain. M, 100 bp DNA ladder; Lane 1 4, isolate; Lane 5, positive control of commercial kit.

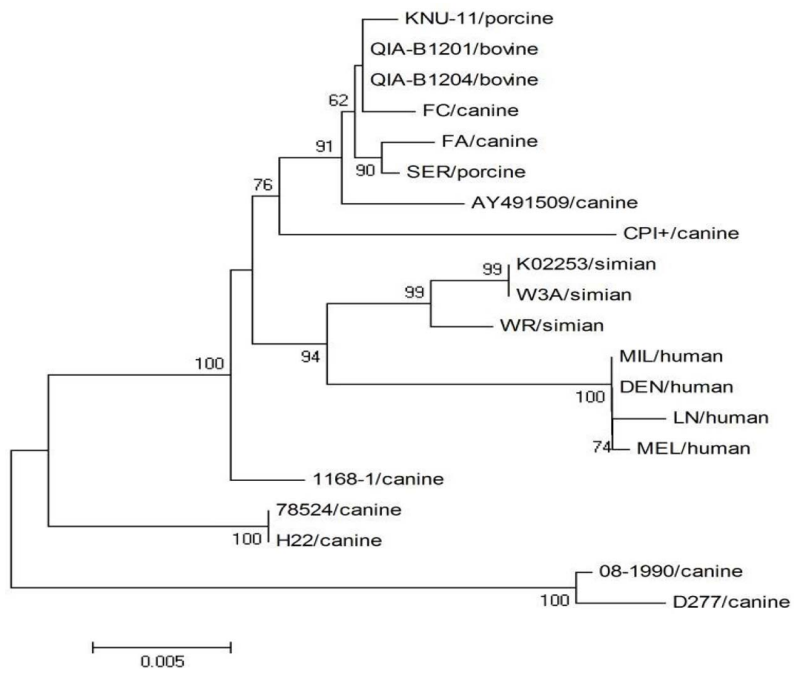

Fig. 3. Phylogenetic analysis based on the complete $F$ gene nucleotide sequences of the isolates and other sequences of PIV5s obtained from GenBank database.

with $810,630,594$ bp in size on $1.8 \%$ agarose gel. A total of 1656 bp nucleotide sequences of the complete F gene encoding fusion protein from two isolates were determined and their amino acids sequences were deduced. The $\mathrm{F}$ gene sequences of 18 other PIV5 obtained from GenBank were compared with those of the two isolates to analyze molecular epidemic relationships. The nucleotide sequence analysis showed that two isolates had high similarity with other human and animal PIV5 isolates ranging from 98.1 to $99.8 \%$

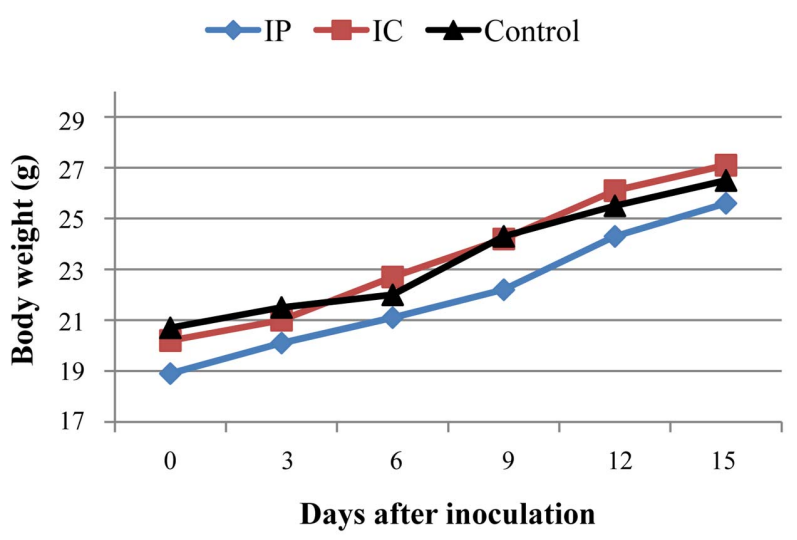

Fig. 4. Change of body weight in mice inoculated with the bPIV5, QIA-B1201 strain via the intraperitoneal, or intracranial route. They did not show any weight loss for 15 days.

(Fig. 3). The highest sequence similarity of the two isolates corresponded to the KNU-11 strain $(99.8 \%$ at the nucleotide and amino acid level) isolated from suckling piglet in Korea in 2012. The lowest nucleotide similarity of the isolates with non-Korean PIV5 strain was with LN strain (98.0\%) isolated from human. Therefore, nucleotide sequence analysis revealed that the newly isolated viruses were confirmed as bovine PIV5.

\section{Mouse inoculation test}

To investigate the pathogenicity of the bPIV5 isolate, QIAB1201 strain containing $10^{7.0} \mathrm{TCID}_{50} / \mathrm{mL}$ were inoculated into 5 week-old ICR mice via IC $(0.03 \mathrm{~mL})$ and IP $(0.5 \mathrm{~mL})$ route. All mice were observed for 15 days. None of the inoculated mice both with IC or IP showed any symptoms and demonstrated any significantly change of body weight (Fig. 4).

\section{Discussion}

In the present study, we describe the isolation of bPIV5 from cattle with neurological disease. Four viruses were isolated from four brain samples of 70 animals and 12 of 532 brain samples were positive against PIV5 in RT-PCR. Although two brain samples obtained from cattle resided in other farms revealed positive reaction in RT-PCR, we could not isolate PIV5. That may be responsible for the physical properties of PIV5 or low viral titer within the brain samples. The isolation of PIV5 has been reported in a variety of animals such as simian, dog, pig and human, but not in cattle. Previous studies demonstrated that PIV5 originated from several species was cultivated in Vero, MDCK, MDBK, BHK21 and porcine alveolar macrophage (PAM) cells $[1,7$, 13]. The bPIV5 strains in this study also grew well in MDBK, Vero and NG108-15 cells and distinctive CPE in the infected NG108-15 cells was shown within 3 DPI. He et al. [6] reported that PIV5 SH protein plays a key role in viral pathogenesis and its gene has a relation to induction of apo- 
ptosis through the TNF- $\alpha$ mediated extrinsic apoptotic pathway. The HA and HAD activity of PIV5 has been reported using several kinds of red blood cells [1]. In our study, bPIV5, QIA-B1201 agglutinated pig, guinea pig, chicken, mice and goose erythrocytes at $4^{\circ} \mathrm{C}$ and room temperature and showed high HA titer when the chicken erythrocytes were used at $4^{\circ} \mathrm{C}$, indicating that HA activity has strong interaction between ligand of bPIV5 and receptor of chicken erythrocytes.

Sequence comparison of several species containing human, canine and porcine revealed a surprising similarity at both the nucleotide and amino acid levels (97 100\%), even though the viruses had been isolated over 30 years and originated from countries around the world [4]. Until recently, several PIVs have been isolated only from the respiratory samples $[1,7]$. Comparing the nucleotide sequence similarity of $\mathrm{F}$ gene with PIV5 isolated from several species, the bPIV5 (QIA-B1201 and QIA-B1204) formed a close phylogenetic relationship with the KNU-11 strain, which was isolated from a suckling piglet in Korea and with FC strain isolated from dog in China. However, it was a little distantly related to other PIV5 strains (D277 and 08-1990 strain) originated from canine. In the phylogenetic tree, even though the monkey originated (W3A, WR and K02253) viruses and human viruses (DEN, LN, MIL and MEL) formed a clade and animal originated (porcine, canine and bovine) viruses formed other clade, it seems that there is no big difference in nucleotide similarity among the canine/porcine/bovine/monkey and human PIV5.

Although it is still not clear whether the bPIV5 is cordially connected to induction of neurologic symptoms or can cause clinical disease alone, this virus was isolated from brain samples showing downer cattle syndrome. PIV5 alone or mixed infection with canine viruses and bacterial pathogens such as Bordetella bronchiseptica resulted in high morbidity and mortality [3]. Previous researcher reported that coinfection with viral or environmental factors may contribute to the virulence of pPIV5 exacerbating the clinical symptoms of disease [13]. In addition, it is also noted that children with human parainfluenza virus infection had brain legions compatible with acute necrotizing encephalopathy [14]. As mentioned above, bPIV5 alone or mixed with bacterial or viral pathogens such as Clostridium botulinum, BVDV, BRSV may also have acted as trigger for neurologic symptoms in cattle.

In conclusion, based on the biological method we characterized bPIV5 causing distinctive CPE in several continuous cell lines and we proved HAD and HA activity of PIV5 with several kinds of animal erythrocytes. The $\mathrm{F}$ gene of the Korean bPIV5 isolates (QIA-B1201 and QIA-B1204) was fully sequenced in order to investigate molecular characteristics. Nucleotide analysis demonstrated that bPIV5 had a close similarity with KUN-11 strain. Furthermore, mouse experiment indicated that bPIV5 does not cause any clinical symptoms in 5 week-old mice inoculated by IC and IP route. We did not elucidate pathogenicity of bPIV5 in mice. Therefore, further studies would be required to determine if the bPIV5 can cause neurologic symptoms in cattle and develop new vaccine against bPIV5.

\section{Acknowledgments}

This work was financially supported by a grant from Korean Institute of Planning and Evaluation for Technology in Food, Agriculture, Forestry and Fisheres (iPET).

\section{References}

1. Ajiki M, Takamura K, Hiramatsu K, Nakai M, Sasaki N, Konishi S. Isolation and characterization of parainfluenza 5 virus from a dog. Nihon Juigaku Zasshi 1982, 44, 607-618.

2. Appel MJ, Percy DH. SV-5-like parainfluenza virus in dogs. J Am Vet Med Assoc 1970, 156, 1778-1781.

3. Buonavoglia C, Martella V. Canine respiratory viruses. Vet Res 2007, 38, 355-373.

4. Chatziandreou N, Stock N, Young D, Andrejeva J, Hagmaier K, McGeoc DJ, Randall RE. Relationships and host range of human, canine, simian and porcine isolates of simian virus 5 (Parainfluenza virus 5). J Gen Virol 2004, 85, 3007-3016.

5. Goswami KKA, Lange LS, Mitchell DN, Cameron KR, Russell WC. Does simian virus 5 infect humans? J Gen Virol 1984, 65, 1295-1303.

6. He B, Lin GY, Durbin JE, Durbin RK, Lamb RA. The SH integral membrane protein of the paramyxovirus simian virus 5 is required to block apoptosis in MDBK cells. $\mathrm{J}$ Virol 2001, 75, 4068-4079.

7. Heinen E, Herbst W, Schmeer N. Isolation of a cytopathogenic virus from a case of porcine reproductive and respiratory syndrome (PRRS) and its characterization as parainfluenza virus type 2. Arch Virol 1998, 143, 22332239.

8. Hsiung GD. Parainfluenza-5 virus. Infection of man and animal. Prog Med Virol 1972, 14, 241-274.

9. Hull RN, Minner JR, Smith JW. New viral agents recovered from tissue cultures of monkey kidney cells. I. Origin and properties of cytopathogenic agents S.V.1, S.V.2, S.V.4, S.V.5, S.V.6, S.V.11, S.V.12 and S.V.15. Am J Hyg 1956, 63, 204-215.

10. Johnson JB, Capraro GA, Parks GD. Differential mechanisms of complement-mediated neutralization of the closely related paramyxoviruses simian virus 5 and mumps virus. Virology 2008, 376, 112-123.

11. Lamb RA, Parks GD. Paramyxoviridae: the viruses and their replication. In: Knipe DM, Howley PM (eds.). Fields Virology. 5th ed. pp. 1449-1496, Lippincott WIlliams \& Wilkins, Philadelphia, 2007.

12. Larkin MA, Blackshields G, Brown NP, Chenna R, McGettigan PA, McWilliam H, Valentin F, Wallace IM, Wilm A, Lopez R, Thompson JD, Gibson TJ, Higgins DG. Clustal $\mathrm{W}$ and Clustal $\mathrm{X}$ version 2.0. Bioinformatics 2007, 23, 2947-2948.

13. Lee YN, Lee C. Complete genome sequence of a novel 
porcine parainfluenza virus 5 isolate in Korea. Arch Virol 2013, 158, 1765-1772.

14. Mastroyianni SD, Voudris KA, Katsarou E, Gionnis D, Mavromatis P, Vagiakou EA, Skardoutsou A. Acute necrotizing encephalopathy associated with parainfluenza virus in a Caucasian child. J Child Neurol 2003, 18, 570572.

15. Ravid D, Leser GP, Lamb RA. A role for caveolin 1 in assembly and budding of the paramyxovirus parainfluenza virus 5. J Virol 2010, 84, 9749-9759.

16. Schmitt AP, He B, Lamb RA. Involvement of the cytoplasmic domain of the hemagglutinin-neuraminidase protein in assembly of the paramyxovirus simian virus 5. J Virol 1999, 73, 8703-8712.

17. Sun D, Luthra P, Xu P, Yoon H, He B. Identification of a phosphorylation site within the $\mathrm{P}$ protein important for mRNA transcription and growth of parainfluenza virus 5. J Virol 2011, 85, 8376-8385.

18. Tamura K, Dudley J, Nei M, Kumar S. MEGA4: molecular evolutionary genetics analysis (MEGA) software version 4.0. Mol Biol Evol 2007, 24, 1596-1599.

19. Wilson RL, Fuentes SM, Wang P, Taddeo EC, Klatt A, Henderson AJ, He B. Function of small hydrophobic proteins of paramyxovirus. J Virol 2006, 80, 1700-1709. 